\title{
Non-assisted versus neuro-navigated and XperCT-guided external ventricular catheter placement: a comparative cadaver study
}

\author{
Oliver P. Gautschi • N. R. Smoll • M. Kotowski • \\ B. Schatlo • M. Tosic • B. Stimec • J. Fasel • K. Schaller • \\ P. Bijlenga
}

Received: 30 November 2013 / Accepted: 30 January 2014 / Published online: 25 February 2014

(C) Springer-Verlag Wien 2014

\begin{abstract}
Background and purpose Accurate placement of an external ventricular drain (EVD) for the treatment of hydrocephalus is of paramount importance for its functionality and in order to minimize morbidity and complications. The aim of this study was to compare two different drain insertion assistance tools with the traditional free-hand anatomical landmark method, and to measure efficacy, safety and precision.

Methods Ten cadaver heads were prepared by opening large bone windows centered on Kocher's points on both sides. Nineteen physicians, divided in two groups (trainees and board certified neurosurgeons) performed EVD insertions. The target for the ventricular drain tip was the ipsilateral foramen of Monro. Each participant inserted the external ventricular catheter in three different ways: 1) free-hand by anatomical landmarks, 2) neuronavigation-assisted $(\mathrm{NN})$, and 3) XperCT-guided (XCT). The number of ventricular hits and dangerous trajectories; time to proceed; radiation exposure of patients and physicians; distance of the catheter tip to target
\end{abstract}

O. P. Gautschi $(\bowtie) \cdot$ N. R. Smoll • M. Kotowski • B. Schatlo •

K. Schaller $\cdot$ P. Bijlenga

Service de Neurochirurgie, Département de Neurosciences cliniques, Faculté de Médecine, Hôpitaux Universitaires de Genève, Geneva,

Switzerland

e-mail: ogautschi@gmail.com

B. Schatlo

Department of Neurosurgery, Georg-August-University of Göttingen, Göttingen, Germany

M. Tosic

Institut de Radiophysique, Centre Hospitalier Universitaire Vaudois, Lausanne, Switzerland

B. Stimec $\cdot$ J. Fasel

Anatomie clinique, Départment de physiologie cellulaire et métabolisme, Faculté de Médecine, Geneva, Switzerland and size of deviations projected in the orthogonal plans were measured and compared.

Results Insertion using XCT increased the probability of ventricular puncture from 69.2 to $90.2 \%(p=0.02)$. Non-assisted placements were significantly less precise (catheter tip to target distance $14.3 \pm 7.4 \mathrm{~mm}$ versus $9.6 \pm 7.2 \mathrm{~mm}$, $p=0.0003$ ). The insertion time to proceed increased from $3.04 \pm 2.06 \mathrm{~min}$. to $7.3 \pm 3.6 \mathrm{~min}$. $(p<0.001)$. The $\mathrm{X}$-ray exposure for XCT was $32.23 \mathrm{mSv}$, but could be reduced to $13.9 \mathrm{mSv}$ if patients were initially imaged in the hybridoperating suite. No supplementary radiation exposure is needed for $\mathrm{NN}$ if patients are imaged according to a navigation protocol initially.

Conclusion This ex vivo study demonstrates a significantly improved accuracy and safety using either NN or XCTassisted methods. Therefore, efforts should be undertaken to implement these new technologies into daily clinical practice. However, the accuracy versus urgency of an EVD placement has to be balanced, as the image-guided insertion technique will implicate a longer preparation time due to a specific image acquisition and trajectory planning.

Keywords External ventriculardrain · Accuracy $\cdot$ Anatomical landmarks $\cdot$ Neuronavigation $\cdot$ Real-time insertion technique

\section{Introduction}

The positioning of an external ventricular drain (EVD) belongs to the most commonly performed neurosurgical procedures, often performed by neurosurgical residents [12]. It can be accomplished either as a bedside procedure or in the operating room, usually under time-sensitive conditions [21]. Numerous indications exist for EVD placement; most frequent among them is hydrocephalus due to subarachnoid, 
intraventricular, or intraparenchymal hemorrhage. Other reasons include traumatic brain injury in order to measure intracranial pressure (ICP) and/or to release cerebrospinal fluid (CSF), CSF obstruction, either congenital or acquired, or, infection or dysfunction of an already implanted shunt system, or EVD placement for fibrinolytic clot lysis in case of intraventricular hemorrhage $[5,18]$. The ipsilateral frontal horn, including the tip of the 3rd ventricle, is regarded as an optimal location of the catheter tip [12]. The percentage of EVD catheters with a suboptimal location is in the range of 6 to $22 \%[10,17]$. In patients with very narrow or slit ventricles, the successful introduction of an EVD catheter on the first attempt may be as low as $32 \%$ [15]. Therefore, the accuracy of the ventricular drain placement is of paramount importance to maintain CSF derivation and to minimize morbidity and mortality. Moreover, not only the accuracy, but also time is a crucial factor, because the placement of an EVD is usually a lifesaving procedure. Many ventricular catheter placements, however, still require multiple attempts to obtain a successful CSF drainage, with each placement attempt increasing the risk of rupturing a blood vessel and violating adjacent brain tissue. O'Neill et al., reported in a striking survey of 934 responding practicing neurosurgeons and 100 responding neurosurgery residents that the greatest number of EVD placement attempts reported during a single procedure ranged from 2 to 20 with a mean of 7.3 [20]. Although hemorrhagic complications were observed in $5.7 \%$ in a previously performed meta-analysis, clinically significant hemorrhage (causing new neurological deficit, death, or the need for a subsequent surgical intervention) due to EVD placement was reported in less than $1 \%$ [3]. Another recent meta-analysis using a methodologically more sound analysis with a random-effects model and weighted average calculations revealed a similar overall hemorrhagic complication rate of approximately $7 \%$ and a rate of significant hemorrhage of $0.8 \%$ [2]. An additional important contributing issue in the management of EVDs is the accompanying hazard of CSF infection with a reported range of 0 to $45 \%$ in the current literature [14].

In order to improve the accuracy and subsequent safety of EVD placement, several techniques have been developed, including frame-based stereotactic, frameless navigation, endoscopically assisted, and ultrasound-guided techniques.

The aim of this work was to compare the insertion technique of intraventricular catheters using anatomical landmarks (free-hand technique) with the neuronavigation-assisted (NN), and XperCT-guidance-assisted (XCT) method. The accuracy, procedure duration, safety of insertion, and radiation exposure for patients and surgeons were assessed to measure the effect of complex guidance technologies on ventricular drain placement.

\section{Material and methods}

Ten Formol-fixed human cadaver heads (all specimens were provided by the Geneva University Anatomy Sector as research and teaching material from individuals that donated their body to science and education and handled according to the Swiss Federal Law) were prepared by wide trepanation of the calvaria and excision of the underlying dura mater centered on the Kocher's point on both sides. All cadaver heads had no intracranial pathology apart from cerebral atrophy according to the available medical data. After preparation of the heads, the skin was reclosed with watertight sutures in order to restore a realistic anatomical situation. The skin was re-draped to mask previous skin incisions after each experiment. The entry point was identified and a linear skin incision was performed to start each experiment. The navigated insertion technique was performed using BrainLab VectorVision 2 neuronavigation systems (NN) (BrainLab, Feldkirchen, Germany). The XperCT-guided insertion technique (XCT) was performed using Allura Xper FD20 and XperCT-imaging and XperGuide tools (Philips Healthcare, Best, Netherlands). Nineteen study participants, divided in two groups (trainees and board certified neurosurgeons) inserted the EVDs into frontal horns of both lateral ventricles. The first experiment consisted of: 1) introducing a first catheter using anatomical landmarks, 2) performing a XperCT to acquire the volumetric images to perform the neuronavigation, 3 ) removing the first catheter, 4) co-registrating the neuronavigation, 5) planning the trajectory, 6) inserting a second catheter using the neuronavigation and the navigated stylet type mandrin (BrainLab, Feldkirchen, Germany), 7) defining the trajectory of the third catheter with XperGuide, 8) marking the entry point and orientation of insertion with a laser in the sagittal plan using probe eye view fluoroscopic tracking, 9) under lateral fluoroscopic real-time monitoring, inserting of the catheter with a stylet, and 10) performing a second XperCT to record ventricular drain positions. Each study participant performed the experiment at least two times on two different days. The procedure of the EVD placement using anatomical landmarks was performed according to the technique described by Friedman and Vries [7]. Specifically, after burr hole trepanation over the Kocher point with subsequent opening of the dura and arachnoidea (this was part of the specimen preparation with re-closure of the skin), the EVD catheter was inserted in a free-hand manner directed in the orthogonal planes towards the foramen of Monro using the ipsilateral orbital medial canthus and the tragus of the ipsilateral ear as the two indirect guiding anatomical landmarks.

The following variables were measured and analyzed: (1) frequency of ventricular hits, corresponding to the number of catheters observed with at least one lateral hole within a ventricle per number of insertions, (2) frequency of danger zones hits, corresponding to the number of catheters passing 
through one or more danger zones per number of insertions, and (3) distance to target. Danger zones were defined as internal capsule, thalamus, fornix, cingulum, and interhemispheric fissure. Each danger zone was identified in each specimen by means of XperCT. The distance to target was defined as the distance between the tip of the catheter and the center of the ipsilateral foramen of Monro, measured as vector length (shown in Fig. 1 as a), and length of vector projections on vertical orthogonal plans: anterior to posterior (A-P) (Fig. 1b), lateral to medial (Fig. 1c) and superior to inferior (Fig. 1d). The time of the procedure started when the surgeon was touching the head to identify the anatomical landmarks, to register the neuronavigation or to identify the entry point using the XperCT for non-assisted, NN-assisted and XCTassisted placement, respectively. The measurement stopped when the drain was considered to be in place. The preparation time between starting the XperCT and beginning the registration or entry point identification was measured once for each head. Both average neuronavigation preparation time and XCT-guided method preparation time were added to the drain placement time in order to obtain the procedure time. X-ray radiation dose to the patient (respective to the cadaver head) was measured on both eyes using a thermoluminescent dosimeter (TLD). The radiation doses to the physician were measured using a film badge dosimeter placed on the chest over and under the shielding apron as well as a TLD applied on one leg and on the head and mounted on the ring fingers on both hands.

Three conditions: non-assisted, NN, and XCT-guided drain placement were compared using three study groups: placement by neurosurgical trainees, board certified neurosurgeons, and all placements regardless of their training level. All measurements were performed on XperCT images (ventricular hits, trajectory safety, and distance to target) by two neurosurgeons blinded to the insertion procedure.

Fig. 1 Definition of distance measurements. a Between the tip of catheter and the center of the ipsilateral foramen of Monro measured as vector length (T-T Distance) and the length of vector projections on vertical orthogonal plans on b sagittal plane (Anterior-Posterior), c coronal plane (Lateral-Medial) and d sagittal plane (Superior-Inferior)
Statistical analysis

It is estimated from the literature that multiple drain insertions are necessary to enter the frontal horn of the lateral ventricle in $30 \%$ of EVD interventions [10]. We expected that the use of the NN or XCT-guided method would reduce the number of unsuccessful catheter insertions. We estimated that unsuccessful insertions would be less than $10 \%$. Considering a standard error of $5 \%$ and a one-way test, 49 catheter placements using each method are required to achieve a study power larger than $80 \%$. Therefore, we planned to perform 51 EVD placements using each technique. Results are reported as frequencies of observation. Comparisons between groups are reported as odd ratios with a $95 \%$ confidence interval (CI) and levels of significance were assessed using the Fisher exact test for categorical values. Continuous parametric values are reported as mean and standard error of the mean. The probability of two means to be different is calculated using Student's $t$-test. The significance level used was set at $5 \%$.

\section{Results}

Ventricular hits, trajectory safety, and error distances

Ventricles were hit in $69.2 \%$ of catheter insertions using anatomical landmarks (non-assisted), as compared to $83.3 \%$ with the use of guidance (NN or XCT-guided method taken together) $(p=0.067)$ (Table 1). XCT-assisted insertions of catheters were significantly more likely to be placed in the ventricle than those using anatomical landmarks (OR 4.1, 95\% CI 1.4 12.2, $p=0.012$ ). Difference between both assisting methods was, however, not significant (OR 2.83, 95\% CI 0.92, $8.74, p=0.07)$.

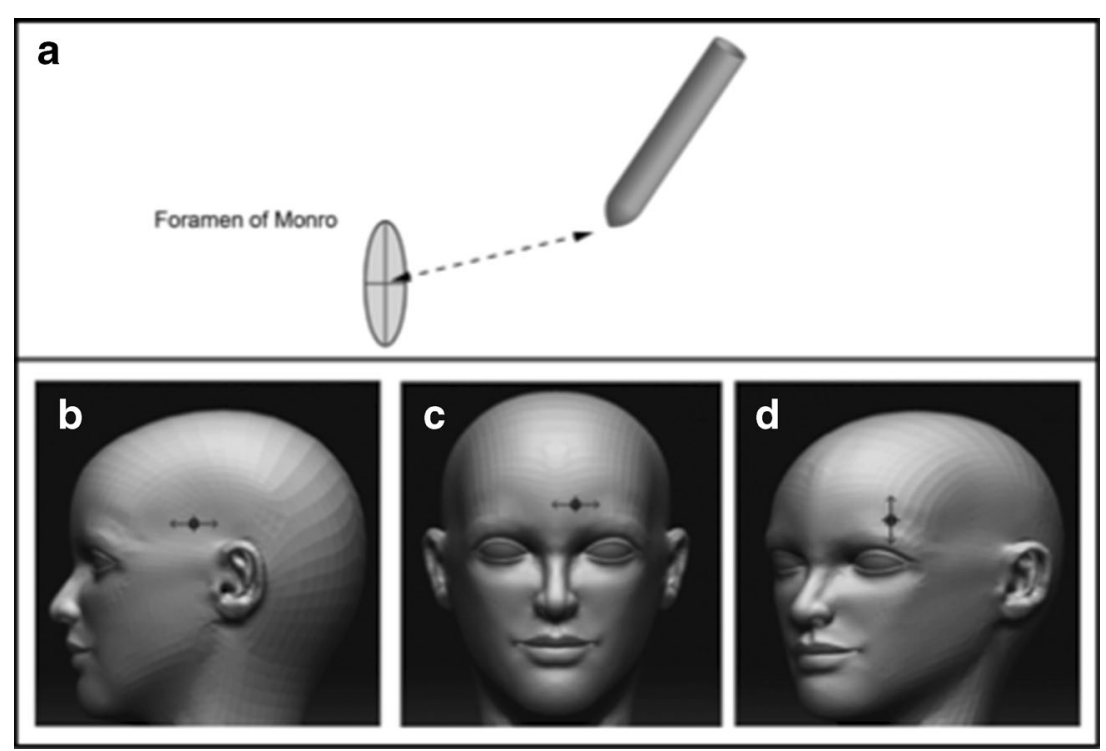


Table 1 Subjects and measures by guidance and level of experience

\begin{tabular}{|c|c|c|c|c|c|}
\hline & Non-assisted & $\begin{array}{l}\text { Neuro-navigation } \\
(\mathrm{NN})\end{array}$ & $\begin{array}{l}\text { XperCT-guided } \\
\text { (XCT) }\end{array}$ & $\begin{array}{l}\text { Assisted } \\
\text { (combined) }\end{array}$ & $\begin{array}{l}p \text {-value (non-assisted } \\
\text { vs. assisted) }\end{array}$ \\
\hline \multicolumn{6}{|l|}{ Experience level } \\
\hline Trainee & 37 & 36 & 36 & 72 & \multirow[t]{2}{*}{$>0.99$} \\
\hline Board Certified & 15 & 15 & 15 & 30 & \\
\hline \multicolumn{6}{|l|}{ Placement location } \\
\hline Extraventricular & 16 & 12 & 5 & 17 & \multirow[t]{2}{*}{0.067} \\
\hline Intraventricular & 36 & 39 & 46 & 85 & \\
\hline Safe trajectory & 31 & 42 & 39 & 81 & 0.013 \\
\hline Kinked catheters & 3 & 1 & 4 & 5 & - \\
\hline Insertion time (mean $\pm \mathrm{SD})$, min. & $3.04 \pm 2.06$ & $6.5 \pm 3.4$ & $8.0 \pm 3.7$ & $7.3 \pm 3.6$ & $<0.0001$ \\
\hline $\mathrm{T}$-T Distance (mean $\pm \mathrm{SD}), \mathrm{mm}$ & $14.3 \pm 7.4$ & $10.1 \pm 7.2$ & $9.1 \pm 7.3$ & $9.6 \pm 7.2$ & $<0.001$ \\
\hline Ant-Post (mean \pm SD), mm & $9.4 \pm 7.8$ & $5.1 \pm 4.7$ & $4.8 \pm 5.2$ & $5.0 \pm 4.9$ & $<0.001$ \\
\hline Lat-Med (mean $\pm \mathrm{SD}), \mathrm{mm}$ & $5.0 \pm 4.1$ & $3.8 \pm 4.5$ & $4.3 \pm 5.0$ & $4.1 \pm 4.7$ & 0.43 \\
\hline Sup-Inf (mean \pm SD), mm & $6.6 \pm 5.1$ & $5.4 \pm 6.5$ & $3.9 \pm 5.0$ & $4.6 \pm 5.8$ & 0.04 \\
\hline Total Insertions & 52 & 51 & 51 & 102 & - \\
\hline
\end{tabular}

Unsafe trajectories were observed after $40.4 \%$ of nonassisted placements in contrast to $20.6 \%$ of assisted insertions $(p=0.013)$. Attempts with non-assisted placement methods were more likely to result in an unsafe trajectory compared to those using $\mathrm{NN}$ (OR 3.16, 95\% CI 1.27, 7.84, $p=0.013$ ). However, no significant difference in final intraventricular trajectory safety comparing XCT-guided placement to the $\mathrm{NN}$-assisted placement method could be revealed (OR 1.44, $95 \%$ CI $0.55,3.78, p=0.46$ ).
Figure 2 demonstrates that the average distance to target is larger in the non-assisted group, but that this error is largest in the anterior to posterior (A-P) direction. Neuronavigation and XCT-guided methods produced smaller errors in the A-P direction, but we should note that the absolute difference is small. The average maximum distance of the catheter tip to the target was $4.2 \mathrm{~mm}$ or $29.3 \%$ smaller $(p=0.002)$ for $\mathrm{NN}$, and $4.6 \mathrm{~mm}$ or $32.2 \%(p=0.001)$ for XCT-guided placements in comparison to the manual placements. The lateral-medial and
Fig. 2 Differences in target precisions compared between non-assisted (None), neuronavigated

(Neuronavigation) and Xper-CT guided (Guided). Distances (Average and $95 \% \mathrm{CI}$ ) measured as defined in Fig. 1

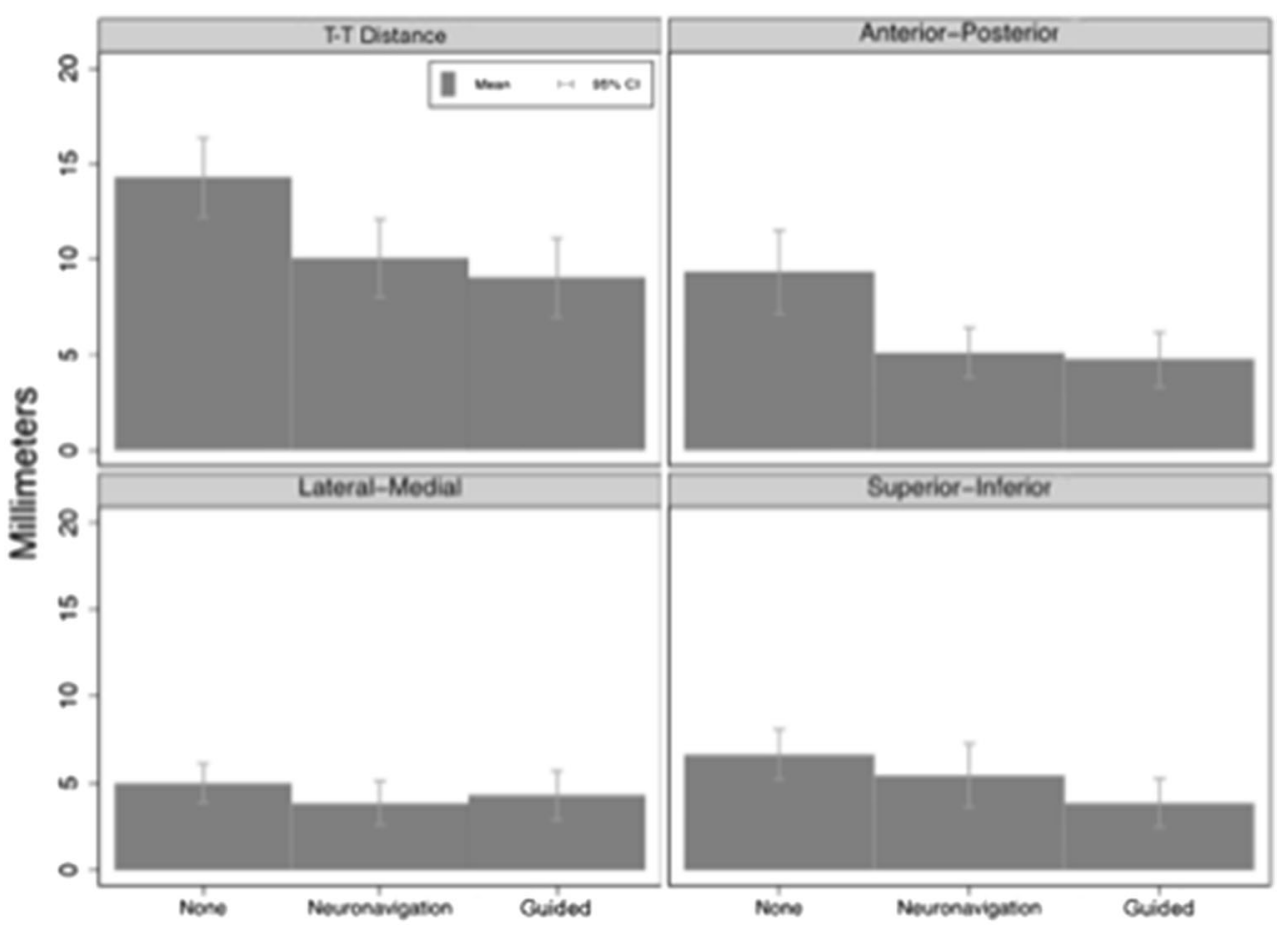


superior-inferior planes did not demonstrate any strong differences in means (see Fig. 2).

During the course of the experiment, it was observed that the feedback sound during the $\mathrm{NN}$-assisted method when hitting the target was inducing a small back-off reflex that resulted in placement of the drain that was a few millimeters more superficial than scheduled. We corrected this by removing the sound but too late in the study to observe a significant improvement.

\section{Catheter kinking}

During the course of the experiment, we observed the phenomenon of a catheter kinking during insertion (Fig. 3). This was easily recognized during the XCT-guided method but also observed on images performed after drain placement. The correct use of the NN stylet prevented this phenomenon. Out of 51 punctures performed using a NN stylet, only one kinking was observed (with observed incorrect use thereof). In contrast eight kinkings were observed among 103 drain placements using traditional EVD stylets $(p=0.27)$. All kinked drains were inserted by junior residents. Only two out of eight kinked drains had a catheter hole in contact with the ventricle. The most distal part of kinked drains was significantly more distant from the target than the tip of non-kinked catheters $(21.11 \pm 8.5 \mathrm{~mm}$ versus $10.6 \pm 7.2 \mathrm{~mm}, p<0.001)$.

When excluding the kinked drains from the analysis, $71.4 \%$ catheters were inserted in the ventricles using anatomical landmarks and $86.6 \%$ when using assisted methods ( $p=$ 0.04). Catheters placed using the XCT-guided method were more likely intraventricular than catheters placed using $\mathrm{NN}$ (OR 6.35, 95\%CI 1.3, 30.4; $p=0.021$ ). The average maximum distance of the catheter tip to the target was $4.15 \mathrm{~mm}$ or $29.9 \%$ smaller $(p=0.004)$ for $\mathrm{NN}$, and $5.65 \mathrm{~mm}$ or $40.7 \%(p=$ 0.0001 ) for XCT-guided placements, as compared to manual placements. Neuronavigation and XCT-guided methods were more accurate than non-assisted insertion in the A-P direction

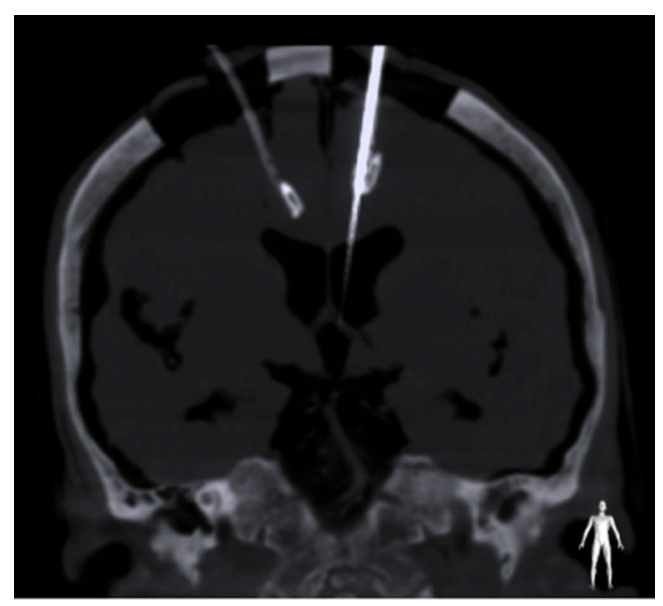

Fig. 3 Example of a catheter kinking during insertion $(p<0.01)$. The XCT-guided method was more accurate than both other methods in the superior-inferior direction $(p<0.001)$. Precision was similar for all methods in the lateral-medial plane.

Time to completion

The time to insert the catheter was shorter using manual placement. Manual placement was faster than neuronavigation by $3.5 \mathrm{~min}$ (difference in means, $p<0.001$ ) and XCT-guided method by $5 \mathrm{~min}$ (difference in means, $p<0.001$ ). The average $\mathrm{NN}$ planning time was $3.8 \pm 3.7 \mathrm{~min}$ and $\mathrm{NN}$ registration $3.9 \pm 2.3 \mathrm{~min}$.

Considering the registration for XCT-guidance is made with an image acquisition and the planning is performed in parallel to the neuronavigation planning using the Xper-Guide tool, no planning time was measured. It was, however, consistently longer than the neuronavigation planning time but shorter than the neuronavigation planning and registration time combined. It can be estimated to be shorter than $10 \mathrm{~min}$ in $95 \%$ of the cases.

\section{Radiation dose}

The different total average radiation doses to the patient, respectively towards the human cadaver heads are depicted in Table 2. The non-assisted placement method (free-hand by anatomical landmarks) does not require supplementary scans other than the initial normal head CT scan leading to the diagnosis and subsequent treatment decision. For the NNassisted method, however, a new head CT scan with fine cuts is a requirement if the data cannot be generated from the initial diagnostic imaging, leading to a median radiation dose of $18.36 \mathrm{mSv}$, which is the equivalent of a mean background radiation of around 4.5 years. For the XCT-guided method (with fluoroscopy guidance), however, an additional fine cut head CT scan acquired on site is mandatory. Additionally, the method itself is performed under radiographic guidance, leading to a mean total dose of $32.23 \mathrm{mSv}$ per procedure, which corresponds to a mean background radiation of around eight years. A subanalysis of the tracked procedures performed by certified surgeons revealed a substantial reduction of the mean total dose per procedure of $25.86 \mathrm{mSv}$, which corresponds to a mean background radiation of around six years. There was, of course, no reduction of the total dose per procedure for the $\mathrm{NN}$-assisted method, because the necessary irradiation was not influenced by the level of experience of the performer.

The mean radiation dose to the surgeon, who performed the procedure with the XCT-guided method was also measured, revealing a mean total radiation exposure time of $145 \mathrm{~s}$ per procedure with a mean total radiation dose of $0.0449 \mathrm{mSv}$ to the non-protected body area (corresponds to four days 
Table 2 Radiation doses using Xper-CT guidance (XCT)

\begin{tabular}{llllll}
\hline & Head Xper-CT & \multicolumn{2}{l}{ XperCT-guidance } & & Total dose \\
\cline { 3 - 5 } & $\mathrm{mSv}$ & $\begin{array}{l}\text { Time } \\
\mathrm{sec}\end{array}$ & $\begin{array}{l}\text { Dose/min } \\
\mathrm{mSv} / \mathrm{min}\end{array}$ & $\begin{array}{l}\text { Dose } \\
\mathrm{mSv}\end{array}$ & $\mathrm{mSv}$ \\
\hline Non-assisted & $0-18.36$ & - & - & - & $0-18.36$ \\
Neuronavigated & 18.36 & - & - & - & 18.36 \\
Tracked & 18.36 & 145 & 4.8 & 13.9 & 32.23 \\
Tracked by certified surgeon & 18.36 & 88.5 & 4.8 & 7.5 & 25.86 \\
\hline
\end{tabular}

background radiation). No measurable doses to the lead apron protected area were recorded. The Thermoluminescent Detector (TLD) apposed on the head of the surgeon measured an average dose of $0.0389 \mathrm{mSv}$. It corresponds to four days of background radiation but exposes unprotected eyes to a significant risk of cataract after more than 3,800 procedures or 125 procedures per year during 30 years. The doses measured on the manipulating hand were $1.113 \mathrm{mSv}$ corresponding to four months of background radiation and limits the maximum number of procedures performed per year to less than 450 according to Swiss laws. The doses measured on the feet of the surgeon were $0.022 \mathrm{mSv}$ corresponding to two days of background radiation.

\section{Discussion}

This study shows that the yield and safety of non-assisted intraventricular catheter insertion is poor and can be improved using navigation or guidance. Our observation confirms the many previously reported bad yields of ventricles puncture using solely anatomical landmarks $[10,19,30,31]$. The use of a NN or XCT-guided method improved the yield of intraventricular catheters placement, but more obviously when using the XCT-guided method. A safe trajectory was observed in $59.6 \%$ of the non-assisted insertions compared to $79.4 \%$ in assisted trajectories.

Observations are consistent with the rates reported in Kakarla's et al., study, in which $77 \%$ of free-hand technique drain placements were optimal in the ipsilateral frontal horn and the third ventricle through the foramen of Monro [12]. However, Huyette et al., reported an accurate catheter tip placement in the ipsilateral lateral ventricle in only $56.1 \%$ of the catheter tips and that of a placement in the third ventricle of $8.2 \%$, summing up to a percentage of $64.3 \%$, also using a free-hand pass technique [10]. Notwithstanding, many centers still use the free-hand technique using surface anatomical landmarks.

That assisted methods may increase accuracy has been shown in different studies. One of the largest of singlesurgeon experience was published by Sampath et al., where 70 patients with a mean age of 44.6 years underwent 52 ventriculo-peritoneal (VP) shunt and 18 Ommaya reservoir placement procures using stereotactic neuronavigation with rigid cranial fixation [24]. The authors reported a malposition rate of only $1.4 \%$. Furthermore, there was no proximal malfunction or chemotherapy complication during the mean follow-up of 16.3 months. Stieglitz et al., showed in a pilot study using BrainLab neuronavigation systems a good accuracy of the catheter tip with a mean deviation of the catheter tips from the planned position of $1.5 \mathrm{~mm}$ (range 1.0-2.1 mm) [25]. The authors concluded that the time for planning and installation of the navigation system and placement instrument did not exceed $15 \mathrm{~min}$. Whitehead et al., on the other hand showed an accurate placement of CSF shunt ventricular catheters in a pediatric population using real-time ultrasound guidance [29]. Also Jakola et al., just recently presented their results with a similar technique using a three-dimensional ultrasound-guided placement of ventricular catheters [11]. The authors report an optimal placement of the catheter in a single pass in all four patients. In addition, Thomale et al., presented a smartphone-assisted guide for the placement of ventricular catheters, which has been successfully applied in 35 patients [26]. On the other hand, Mahan et al., recently presented the feasibility and accuracy of electromagnetic image guidance for EVD placement in the intensive care unit (ICU) including 35 patients [17]. Although the use of this technology added approximately $36 \mathrm{~min}$ to the total procedure, it added only $4 \mathrm{~min}$ of additional operative time. There were no unacceptably placed EVD catheters and only two were not perfectly placed in the ipsilateral frontal horn. Hermann et al., reported their results using the same technique (electromagnetic-guided neuronavigation) with 26 children with hydrocephalus and difficult ventricular anatomy or slit ventricles [9]. There were no operative complications and all children had an accurate shunt placement. Already before, Azeem and Origitano had presented their 1-year clinical experience in a consecutive pediatric and adult series of 34 catheters that were placed using an electromagnetic frameless neuronavigation system [1]. Although the authors did not directly evaluate shunt accuracy with postoperative imaging, they reported no proximal shunt failures. Keric et al., published the results of a feasibility study with an image-guided implantation of a pre-calibrated stylet in the ICU [13]. All catheters implanted in the ICU $(n=11)$ were rated as optimally 
positioned ( $<3 \mathrm{~mm}$ deviation from the scheduled trajectory). The authors also reported the mean time for catheter placement in the ICU, which was $30.0 \pm 11.3 \mathrm{~min}$ and the time for the trajectory planning of $12.6 \pm 6.85 \mathrm{~min}$. Villavicencio, et al. published a comparison of revision rates following endoscopically versus nonendoscopically placed ventricular shunt catheters [28]. They found that neuroendoscopy did not independently affect the risk of a subsequent shunt failure. However, an endoscopic shunt placement decreased the odds of a proximal obstruction.

A recently published clinical study deserves specific attention. Wilson et al., compared the accuracy of three methods of ventricular catheter placement including 249 patients using the free-hand technique with anatomical landmarks, ultrasonic guidance, and stereotactic neuronavigation [30]. Ninety-four (55\%) of 170 free-hand passed catheters were placed accurately, compared to 45 ( $88 \%$ ) of 51 stereotactic-guided catheters and 25 (89\%) of 28 ultrasound-guided catheters. The authors found a statistically significant difference between free-hand catheters and stereotactic-guided catheters, as well as between free-hand catheters and ultrasound-guided catheters. Furthermore, the authors could show that the use of stereotactic neuronavigation and ultrasonic guidance reduced proximal shunt failure rates in comparison with the free-hand method using anatomical landmarks.

Also in our study, although a cadaveric experimental study, we observed that the use of an assisted method increased the likelihood to place the catheter tip in the ventricle according to a safer trajectory. The accuracy of the placement of the tip of the catheter was better using an assisted method but remained, nevertheless, off by $10.1 \mathrm{~mm}$ and $9.1 \mathrm{~mm}$ using NN or XCTguided assistance, respectively. The catheters placed using $\mathrm{NN}$-assistance were systematically not implanted deep enough. The use of a warning sound when reaching the target using the NN-assisted method induced a back off reflex probably responsible for some of the too short catheter implantations. Correction by removing the warning sound was applied too late during the course of the study to show a statistically significant improvement.

Catheter kinking was observed during eight out of 154 catheters insertions (5.2\%) and mostly observed when using standard stylet and only observed once during the incorrect use of the neuronavigated stylet. The kinked catheters were significantly more distant to the target than non-kinked catheters $(p<0.001)$. The use of classical stylets and manipulating the drain over the stylets showed, in our cadaveric model, a significant risk of drain kinking that could represent a high risk of hemorrhage in patients. The stiffer consistence of the cadaver brain fixed in Formol may have exaggerated this phenomenon. Nevertheless, the use of either a shorter drain on the standard stylet (with avoiding stretching of the elastic drain), or the use of a stylet with a handle successfully prevented this issue.
As is illustrated in Fig. 3, the cadaveric brains were atrophic and the CSF was predominately replaced by air. Although imaging was performed in the same position as catheter insertion, some degree of brain shift may have occurred during drain placement and explain a higher degree of inaccuracy than previously reported. The same phenomenon could be responsible for the better accuracy using the XCT-guided method as compared to the $\mathrm{NN}$-assisted method. The ability to visualize, 1) the target faintly and 2) the progression of the catheter, allowed correcting of the insertion depth. It also allowed identifying the beginning of a putative catheter kinking.

The procedure time for any assisted method is certainly much longer and technical burden higher than for a free-hand anatomical landmark-based bedside ventriculostomy. Although we could show significant differences in catheter insertion time, the absolute differences were always below a few minutes. No doubt that the time measurements were taken in a virtual setting only assessing the insertion time and planning-registration time separately. Nevertheless, it is realistic to design a procedure where a technician is in charge of the neuronavigation while the surgeon prepares the intervention. If the institutional standard is to perform the ventriculostomy in a sterile environment, the time to transfer the patient would be sufficient to set up the navigation and to plan the trajectory. The delay due to registration and navigated insertion would be minimal and probably less than $15 \mathrm{~min}$. Regarding the insertion of an EVD using XCT-guidance, a drastic revision of the patient management procedure could result in both a more precise and quicker intervention.

The treatment by ventricular drainage is mostly the result of a clinical deterioration followed by a cerebral imaging. If selected patients are directly investigated in the hybrid operative-angiographic room, the delay associated with trajectory planning and tracked catheter insertion is largely compensated by the gain in patient transfer between the imaging facility and the treatment room.

Although the accuracy of XCT-guided and NN-assisted ventriculostomies is higher compared to the non-assisted drain insertion method, the amount of irradiation is highest for the XCT-guided procedure, followed by the $\mathrm{NN}$-assisted method and the non-assisted placement method. The X-ray exposition of XCT-guided drain insertion can be reduced to the tracking time only, if the diagnostic imaging is performed in the hybrid room, but it can be avoided for the $\mathrm{NN}$-assisted procedure if the initial head CT scan is acquired using millimetric slices and no gentry rotation.

There are several limitations of the present study design. First, the cadaver heads were used several times. The calvarian trepanation and dura opening was performed before the experiment. For all subsequent experiments, the study participant started with only a skin incision and subsequent introduction of the EVD catheter through the prepared opening. As 
the study goal was to investigate the EVD positioning and not burr hole preparation, we accepted this small methodological error. Second, we did not measure the specific time for image data acquisition and trajectory planning for the navigated procedures. However, as we know from the study of Mahan et al., the image-navigation technology added approximately $36 \mathrm{~min}$ to the time from when the need was identified to when successful drainage was achieved [17]. Therefore, time to CSF drainage becomes more crucial than perfect accuracy in an urgent situation, where CSF drainage is immediately necessary without compromising the patient's health.

Although the free-hand technique to position an EVD catheter is still the most frequently used, there is definitively space for improvement to optimize the yield of catheter tips inserted in the foramen of Monro. The present results show that NN- or XCT-assisted guidance would have a rather small effect in improving the likelihood to puncture large or extralarge ventricles but might be of benefit in patients with rather small ventricles or deformed ventricular anatomy although not formally investigated in our study $[4,8,9,16,22,32]$. The ultimate goal has to be an optimal EVD placement tailored to the individual patient at the first attempt, which finally may decrease procedure-related morbidity including infection, revision rate, complications, and lengths of stay, as well as mortality $[6,17,23,27,30]$.

\section{Conclusion}

This ex vivo study demonstrates an improved accuracy using either assisted neuronavigation or XperCT-guided methods. Therefore, efforts should be undertaken to implement these new technologies into the daily clinical practice, specifically for patients with rather small ventricles, where the accuracy is more important. However, the accuracy versus urgency of an EVD placement has to be balanced, as the image-guided insertion technique will implicate a longer preparation time due to a specific image acquisition and trajectory planning.

Acknowledgments The authors would like to thank all participating surgeons: S. Momjian, Y. El Hassani, I. Radovanovic, M. Jägersgerg, H. Becker, A. Bartoli, A. Kurzburch, M. Seek, A. Reverdin, and B. Rilliet for their valuable contribution. Special thanks also to Mr T. Gerken and Mrs L. Slegers from Brainlab and Philips for their expertise.

Conflict of interest None.

\section{References}

1. Azeem SS, Origitano TC (2007) Ventricular catheter placement with a frameless neuronavigational system: a 1-year experience. Neurosurgery 60:243-248
2. Bauer DF, Razdan SN, Bartolucci AA, Markert JM (2011) Metaanalysis of hemorrhagic complications from ventriculostomy placement by neurosurgeons. Neurosurgery 69:255-260

3. Binz DD, Toussaint LG 3rd, Friedman JA (2009) Hemorrhagic complications of ventriculostomy placement: a meta-analysis. Neurocrit Care 10:253-256

4. Clark S, Sangra M, Hayhurst C, Kandasamy J, Jenkinson M, Lee M, Mallucci C (2008) The use of noninvasive electromagnetic neuronavigation for slit ventricle syndrome and complex hydrocephalus in a pediatric population. J Neurosurg Pediatr 2:430-434

5. Dey M, Jaffe J, Stadnik A, Awad IA (2012) External ventricular drainage for intraventricular hemorrhage. Curr Neurol Neurosci Rep 12:24-33

6. Dickerman RD, McConathy WJ, Morgan J, Stevens QE, Jolley JT, Schneider S, Mittler MA (2005) Failure rate of frontal versus parietal approaches for proximal catheter placement in ventriculoperitoneal shunts: revisited. J Clin Neurosci 12:781-783

7. Friedman WA, Vries JK (1980) Percutaneous tunnel ventriculostomy. Summary of 100 procedures. J Neurosurg 53:662-665

8. Gil Z, Siomin V, Beni-Adani L, Sira B, Constantini S (2002) Ventricular catheter placement in children with hydrocephalus and small ventricles: the use of a frameless neuronavigation system. Childs Nerv Syst 18:26-29

9. Hermann EJ, Capelle HH, Tschan CA, Krauss JK (2012) Electromagnetic-guided neuronavigation for safe placement of intraventricular catheters in pediatric neurosurgery. J Neurosurg Pediatr 10:327-333

10. Huyette DR, Turnbow BJ, Kaufman C, Vaslow DF, Whiting BB, Oh MY (2008) Accuracy of the freehand pass technique for ventriculostomy catheter placement: retrospective assessment using computed tomography scans. J Neurosurg 108:88-91

11. Jakola AS, Reinertsen I, Selbekk T, Solheim O, Lindseth F, Gulati S, Unsgard G (2013) Three-dimensional ultrasound-guided placement of ventricular catheters. World Neurosurg S1878-8750(13)01008-5. doi:10.1016/j.wneu.2013.08.021

12. Kakarla UK, Kim LJ, Chang SW, Theodore N, Spetzler RF (2008) Safety and accuracy of bedside external ventricular drain placement. Neurosurgery 63:ONS162-ONS167

13. Keric N, Neulen A, Kantelhardt SR, Giese A (2013) Image-guided implantation of pre-calibrated catheters in the ICU: a feasibility study. Acta Neurochir (Wien) 155:1781-1786

14. Kim JH, Desai NS, Ricci J, Stieg PE, Rosengart AJ, Hartl R, Fraser JF (2012) Factors contributing to ventriculostomy infection. World Neurosurg 77:135-140

15. Krombach G, Ganser A, Fricke C, Rohde V, Reinges M, Gilsbach J, Spetzger U (2000) Virtual placement of frontal ventricular catheters using frameless neuronavigation: an "unbloody training" for young neurosurgeons. Minim Invasive Neurosurg 43: $171-175$

16. Levitt MR, O’Neill BR, Ishak GE, Khanna PC, Temkin NR, Ellenbogen RG, Ojemann JG, Browd SR (2012) Image-guided cerebrospinal fluid shunting in children: catheter accuracy and shunt survival. J Neurosurg Pediatr 10:112-117

17. Mahan M, Spetzler RF, Nakaji P (2013) Electromagnetic stereotactic navigation for external ventricular drain placement in the intensive care unit. J Clin Neurosci 20:1718-1722

18. Morgan T, Awad I, Keyl P, Lane K, Hanley D (2008) Preliminary report of the clot lysis evaluating accelerated resolution of intraventricular hemorrhage (CLEAR-IVH) clinical trial. Acta Neurochir Suppl 105:217-220

19. O'Leary ST, Kole MK, Hoover DA, Hysell SE, Thomas A, Shaffrey CI (2000) Efficacy of the Ghajar Guide revisited: a prospective study. J Neurosurg 92:801-803

20. O'Neill BR, Velez DA, Braxton EE, Whiting D, Oh MY (2008) A survey of ventriculostomy and intracranial pressure monitor placement practices. Surg Neurol 70:268-273 
21. Patil V, Lacson R, Vosburgh KG, Wong JM, Prevedello L, Andriole K, Mukundan S, Popp AJ, Khorasani R (2013) Factors associated with external ventricular drain placement accuracy: data from an electronic health record repository. Acta Neurochir 155:1773-1779

22. Reinertsen I, Jakola AS, Friderichsen P, Lindseth F, Solheim O, Selbekk T, Unsgard G (2012) A new system for 3D ultrasoundguided placement of cerebral ventricle catheters. Int J Comput Assist Radiol Surg 7:151-157

23. Sainte-Rose C, Piatt JH, Renier D, Pierre-Kahn A, Hirsch JF, Hoffman HJ, Humphreys RP, Hendrick EB (1991) Mechanical complications in shunts. Pediatr Neurosurg 17:2-9

24. Sampath R, Wadhwa R, Tawfik T, Nanda A, Guthikonda B (2012) Stereotactic placement of ventricular catheters: does it affect proximal malfunction rates? Stereotact Funct Neurosurg 90:97-103

25. Stieglitz LH, Giordano M, Samii M, Luedemann WO (2010) A new tool for frameless stereotactic placement of ventricular catheters. Neurosurgery 67:ons131-ons 135

26. Thomale UW, Knitter T, Schaumann A, Ahmadi SA, Ziegler P, Schulz M, Miethke C (2013) Smartphone-assisted guide for the placement of ventricular catheters. Childs Nerv Syst 29:131-139

27. Toma AK, Camp S, Watkins LD, Grieve J, Kitchen ND (2009) External ventricular drain insertion accuracy: is there a need for change in practice? Neurosurgery 65:1197-1200

28. Villavicencio AT, Leveque JC, McGirt MJ, Hopkins JS, Fuchs HE, George TM (2003) Comparison of revision rates following endoscopically versus nonendoscopically placed ventricular shunt catheters. Surg Neurol 59:375-380

29. Whitehead WE, Jea A, Vachhrajani S, Kulkarni AV, Drake JM (2007) Accurate placement of cerebrospinal fluid shunt ventricular catheters with real-time ultrasound guidance in older children without patent fontanelles. J Neurosurg 107:406-410

30. Wilson TJ, Stetler WR Jr, Al-Holou WN, Sullivan SE (2013) Comparison of the accuracy of ventricular catheter placement using freehand placement, ultrasonic guidance, and stereotactic neuronavigation. J Neurosurg 119:66-70
31. Woernle CM, Burkhardt JK, Bellut D, Krayenbuehl N, Bertalanffy H (2011) Do iatrogenic factors bias the placement of external ventricular catheters?-a single institute experience and review of the literature. Neurol Med Chir (Tokyo) 51:180-186

32. Woodworth GF, McGirt MJ, Elfert P, Sciubba DM, Rigamonti D (2005) Frameless stereotactic ventricular shunt placement for idiopathic intracranial hypertension. Stereotact Funct Neurosurg 83:12-16

\section{Comments}

Gautschi and colleagues performed an interesting study comparing traditional free-hand anatomical-landmark-based versus navigated and XperCT-guided external ventricular drainage (EVD) placement in ten Formol-fixed human cadaver heads. They found that EVC placement using neuronavigation or XperCT-guidance improved accuracy while the insertion time increased.

EVD placement is a frequently performed procedure in neurosurgery, often in emergency situations. The problem of misplacement of the drainage is well-known and analyzed in the literature. Thus, the results of the present study are not surprising. However, the authors give an exact analysis of the sites of misplacement and of the time needed for each procedure. This may help to decide, which technique could be used in a particular given situation. Apart from the limitations of the study discussed by the authors themselves, we have to consider that especially in emergency situations there is not only the time factor, but also a stress factor of the surgeon and the assisting personnel: I do not think that in such a situation the (ideal) time measurements for navigated procedures of the present cadaver study can be transferred to "real life".

Marcus Reinges

Giessen, Germany 